\title{
Evaluation of human gene variant detection in amplicon pools by the GS-FLX parallel Pyrosequencer
}

Roberta Bordoni ${ }^{\dagger 1}$, Raoul Bonnal ${ }^{\dagger 1}$, Ermanno Rizzi ${ }^{\dagger 1}$, Paola Carrera ${ }^{2,3}$, Sara Benedetti2,3, Laura Cremonesi 2,3, Stefania Stenirri2,3, Alessio Colombo3, Cristina Montrasio ${ }^{3}$, Sara Bonalumi2,3, Alberto Albertini ${ }^{1}$, Luigi Rossi Bernardi ${ }^{1}$, Maurizio Ferrari ${ }^{2,3,4}$ and Gianluca De Bellis*1

\begin{abstract}
Address: ${ }^{1}$ Consiglio Nazionale delle Ricerche, Istituto di Tecnologie Biomediche (CNR-ITB), Via F. Cervi 93, I-20090 Segrate, Italy, ${ }^{2}$ Genomic Unit for the Diagnosis of Human Pathologies, San Raffaele Scientific Institute, Milan, Italy, ${ }^{3}$ Laboratory of Clinical Molecular Biology, Diagnostica e Ricerca San Raffaele S.p.A., Milan, Italy and ${ }^{4}$ Vita-Salute San Raffaele University, Milan, Italy

Email: Roberta Bordoni - bordoni@itb.cnr.it; Raoul Bonnal - bonnal@itb.cnr.it; Ermanno Rizzi - rizzi@itb.cnr.it; Paola Carrera - carrera.paola@hsr.it; Sara Benedetti - benedetti.sara@hsr.it; Laura Cremonesi - cremonesi.laura@hsr.it;

Stefania Stenirri - stenirri.stefania@hsr.it; Alessio Colombo - colombo.alessio@hsr.it; Cristina Montrasio - montrasio.cristina@hsr.it; Sara Bonalumi - bonalumi.sara@hsr.it; Alberto Albertini - alberto.albertini@itb.cnr.it; Luigi Rossi Bernardi - rossibernardi@hotmail.com; Maurizio Ferrari - ferrari.maurizio@hsr.it; Gianluca De Bellis* - gianluca.debellis@itb.cnr.it

* Corresponding author †Equal contributors
\end{abstract}

Published: 8 October 2008

BMC Genomics 2008, 9:464 doi:10.1186/147|-2164-9-464

This article is available from: http://www.biomedcentral.com//47I-2/64/9/464

(c) 2008 Bordoni et al; licensee BioMed Central Ltd.

This is an Open Access article distributed under the terms of the Creative Commons Attribution License (http://creativecommons.org/licenses/by/2.0), which permits unrestricted use, distribution, and reproduction in any medium, provided the original work is properly cited.

\begin{abstract}
Background: A new priority in genome research is large-scale resequencing of genes to understand the molecular basis of hereditary disease and cancer. We assessed the ability of massively parallel pyrosequencing to identify sequence variants in pools. From a large collection of human PCR samples we selected 343 PCR products belonging to 16 disease genes and including a large spectrum of sequence variations previously identified by Sanger sequencing. The sequence variants included SNPs and small deletions and insertions (up to $44 \mathrm{bP}$ ), in homozygous or heterozygous state.
\end{abstract}

Results: The DNA was combined in 4 pools containing from 27 to 164 amplicons and from 8,9 to $50,8 \mathrm{~Kb}$ to sequence for a total of II $10 \mathrm{~Kb}$. Pyrosequencing generated over 80 million base pairs of data. Blind searching for sequence variations with a specifically designed bioinformatics procedure identified 465 putative sequence variants, including 412 true variants, 53 false positives (in or adjacent to homopolymeric tracts), no false negatives. All known variants in positions covered with at least $30 \times$ depth were correctly recognized.

Conclusion: Massively parallel pyrosequencing may be used to simplify and speed the search for DNA variations in PCR products. Our results encourage further studies to evaluate molecular diagnostics applications. 


\section{Background}

The availability of a reference human DNA sequence and high throughput technologies such as automated DNA sequencing has made the identification of sequence variations a key tool in several fields of modern biology. Resequencing of large sets of clinically relevant genes, in order to identify variants, is important for understanding the molecular basis of disease and, consequently, for developing diagnostic tests and identifying drug targets. Thus far, large resequencing projects have used a standard sequencing procedure in which gene fragments are amplified by PCR, purified and subjected individually to Sanger sequencing on both strands $[1,2]$.

New-generation genome sequencing technologies have the potential to simplify this task. These new technologies are based on sequencing-by-hybridization [3], sequencing-by-ligation [4] or sequencing-by-synthesis $[5,6]$. The latter methodology, sequencing-by-synthesis, is implemented in the Genome Sequencer GS-FLX System (454 Life Sciences), which produces several hundred thousand DNA reads of at least $200 \mathrm{bp}$; this is done by monitoring the release of pyrophosphate during the growth of a DNA chain driven by a DNA polymerase [6]. The very high throughput of the instrument is achieved by massively parallel pyrosequencing reactions, which generate a highly redundant representation of the DNA regions under scrutiny.

The GS-FLX has been already employed in bacterial genome sequencing, miRNA discovery, cDNA sequencing, ultra-deep sequencing of PCR amplicons and in other fields of application http://www.454.com/news-events/ publications.asp. In a few papers, the technology has been used to generate detailed pictures of large genomic regions by either multiplexed PCR approaches $[7,8]$ or direct genomic enrichment $[9,10]$. None of these studies, however, included a thorough analysis for known sequence variants. Therefore, in this study, we assessed the performance of massively parallel pyrosequencing in the blind, automated search for sequence variations within pools of PCR-amplified DNA from clinical samples.

\section{Results}

We evaluated the performance of the new pyrosequencing technology of the GS-FLX (454 Life Sciences - Roche) in identifying sequence variants in pools of amplicons from human genomic DNA. We selected 16 genes associated with human genetic diseases (Table 1). Genes ranged in size from 4 to 50 exons and had marked allelic heterogeneity. We obtained PCR-amplified DNA corresponding to these genes from the DNA inventory of San Raffaele Hospital; all DNA samples had previously been sequenced by standard Sanger technique. Overall, 165 amplicons, containing 374 genetic variants, were obtained. These ampli- cons were mixed into four unequal pools (Supplementary Table 1 [see Additional file 2]) to test the ability of the sequencer to handle more or less complex DNA mixtures. Several amplicons were included in more than one pool, but always from different patients (not necessarily with the same sequences); therefore, we analyzed a total of 343 PCR products harboring 429 variants previously confirmed by Sanger sequencing: 350 heterozygous SNPs, 43 homozygous SNPs, 23 deletions from 1 to 44 bp (all but one in heterozygous state), and 13 heterozygous insertions from 1 to $7 \mathrm{bp}$.

Amplicon pools were sequenced following the standard GS-FLX procedure. The large set of reads generated was aligned to the reference genomic sequences, yielding a highly redundant representation of the target regions. Sequence variations were detected in blind, without knowledge of the previously determined genotypes.

\section{Amplicon pools and sequence coverage}

The four pools contained from 27 to 164 of the 343 PCR products (Table 2 [see Additional file 1]). In each pool, equimolar amounts of each PCR product were used. Amplicons ranged from 121 to 569 bp (mean, $315 \mathrm{bp}$ ). The reference genome complexity ranged from nearly 9 $\mathrm{Kb}$ (pool 4) to over $50 \mathrm{~Kb}$ (pool 1), for a total of nearly $110 \mathrm{~Kb}$ to be resequenced. Sequencing with the GS-FLX generated over 373000 reads for over $80.8 \mathrm{Mb}$ of sequence. Blast mapping collected nearly $60 \mathrm{Mb}(73 \%)$ of matched sequences; the remaining $27 \%$ was artifactual, mainly primer dimers, presumably generated during the original amplification reactions in which the amplicons were made. Mean read length was $222 \mathrm{bp}$, well within GSFLX specifications. Pool 4 (the smallest) had a lower average read length (198 bp), possibly due to the over-representation of short amplicons. Overall, 104700 bases $(95,6 \%)$ of the reference sequence was covered at least $30 \times$ depth, considered the minimum necessary for reliable sequence variation detection based on preliminary experiments; 309 of the 343 amplicons were fully covered above this threshold. At a less restrictive $10 \times$ depth of coverage, 319 amplicons were fully covered ( $98,0 \%$ of bases).

\section{Identification of sequence variations}

The redundant representation of input sequences was used to calculate the percentage base calls for every sequence position covered $\geq 30 \times$. For positions with sequence heterogeneity, we considered only those in which the minor allele had an allelic fraction $>20 \%$. Thus, 506 sequence variants were identified, with the smallest pool presenting 48 variants and the largest 221 variants (Table 3 [see Additional file 1]). The variants were classified, on the basis of the sequencing results from forward and reverse reads, into top confident (TC), very confident (VC) or not confident (NC) classes. Altogether, 41 
Table I: Reference information for 16 genes ( 165 amplicons, representing 374 different sequence variants) included in the study

\begin{tabular}{|c|c|c|c|c|c|}
\hline Gene & OMIM & Reference sequence & Reference & Amplicons, $\mathrm{n}$ & Nucleotide variations, $n$ \\
\hline$A B C A 3$ & 610921 & NCBI-NM_00I089 & 14 & 15 & 22 \\
\hline \multirow[t]{4}{*}{$A B C A 4$} & 248200 & NCBI-U88667 & 15 & 51 & 115 \\
\hline & 604116 & & & & \\
\hline & 601718 & & & & \\
\hline & 153800 & & & & \\
\hline \multirow[t]{3}{*}{ CACNAIA } & 141500 & ENSG00000|41837 & 16 & 26 & 39 \\
\hline & 108500 & & & & \\
\hline & 183086 & & & & \\
\hline \multirow[t]{2}{*}{ CFTR } & 219700 & ENSG00000001626 & 17 & 13 & 22 \\
\hline & 277180 & & & & \\
\hline EGR2 & 607678 & ENSG00000I 22877 & $*$ & 4 & 6 \\
\hline FTH & 134770 & NCBI-NM_002032 & 18 & 5 & 10 \\
\hline FTL & 600886 & NCBI-NM_000I46.3 & 19 & 5 & 12 \\
\hline$G J B I$ & 302800 & ENSG00000I69562 & $*$ & 5 & 13 \\
\hline$H B B$ & $604 \mid 31$ & NCBI-NT_009237 & 20 & 2 & 11 \\
\hline IRP2 & 147582 & NCBI-NT_0I0I94 & $*$ & 5 & 16 \\
\hline \multirow[t]{7}{*}{ LAMIN A/C } & 181350 & ENSG00000160789 & 21 & 10 & 36 \\
\hline & $|5900|$ & & & & \\
\hline & 605588 & & & & \\
\hline & 115200 & & & & \\
\hline & 151660 & & & & \\
\hline & 248370 & & & & \\
\hline & 176670 & & & & \\
\hline$M P Z$ & 118200 & ENSG00000I 58887 & $*$ & 5 & 14 \\
\hline \multirow[t]{2}{*}{ PMP22 } & 162500 & ENSG00000109099 & $*$ & 4 & 7 \\
\hline & 118300 & & & & \\
\hline \multirow[t]{2}{*}{ SFTPB } & 267450 & NCBI-M2446I & 14 & 4 & 10 \\
\hline & 265120 & & & & \\
\hline \multirow[t]{2}{*}{ SFTPC } & 267450 & NCBI-J03890 & 14 & 3 & 13 \\
\hline & 610913 & & & & \\
\hline SLC4OAI & 606069 & NM_0I4585 & 23 & 8 & 28 \\
\hline
\end{tabular}

* PCR primers and conditions available on request

variants were classified as NC and not further considered (none corresponded to sequence variations defined by Sanger sequencing). There were 379 TC calls, of which $357(94,2 \%)$ were found to correspond to the known sequences once the data were unblinded; There were also 86 VC calls, of which $55(64,0 \%)$ were correctly called.
Thus, there were altogether 53 miscalls (TC and VC), giving a false-positive rate of $0,05 \%$ for the total 104700 bases covered $\geq 30 \times$. Considering the 393 SNP variations, the percentage agreement was $98,5 \%$ for TC calls (Supplementary Table 2 [see Additional file 2]). In contrast, for 
the 36 insertions and deletions, only $56,4 \%$ of TC and $28,6 \%$ of VC calls agreed with known sequences.

We also assessed the ability of the GS-FLX to identify the 429 sequence variations known by Sanger sequencing. Overall, 17 variants were missed: 15 heterozygous SNPs, 1 deletion and 1 insertion (Table 3 [see Additional file 1]). These 17 variants had all been covered at less than $30 \times$ depth, our cutoff for inclusion in the analysis. Had the coverage threshold been $10 x$, we would have identified seven of these missed variations, but we would have also included seven more miscalls, increasing the number of false positives (Supplementary Table 3 [see Additional file 2]).

In this study, we used a $30 \times$ sequence coverage as the minimum necessary for accurate calling with the GS-FLX. Actual coverage with this instrument exceeded $4000 \times$ in a few cases, and nearly $10 \%$ of the total $110 \mathrm{~Kb}$ sequence had an average coverage depth above 1000x. To understand the importance of coverage depth on the accuracy of the sequence calls, we plotted coverage vs. allelic fraction of the heterozygous variants identified in pool 1 (Figure
1). This analysis showed decreasing variability in allelic fraction with increasing coverage; as coverage increased, the values approached 0.5 , the theoretical allelic fraction for one allele of a biallelic polymorphism.

\section{Impact of homopolymers and large indels}

The 53 false positives (40 deletions, 7 insertions, and 6 heterozygous SNPs) obtained with pyrosequencing were checked by repeat Sanger sequencing, which in all cases confirmed the GS-FLX error. These miscalls were all observed to lie in homopolymers, i.e. stretches of at least three consecutive repeats of the same nucleotide. To understand the role of homopolymers in generating falsepositive miscalls with the GS-FLX, we determined the presence of this sequence pattern in the $110 \mathrm{~Kb} \mathrm{DNA}$ under scrutiny (Table 4 [see Additional file 1]. We identified 6840 homopolymers from 3 to $9 \mathrm{bp}$ in length, for a total of over $23 \mathrm{~Kb}(21 \%$ of the DNA sequenced). Thus, only a very small fraction of homopolymers (53 of 6840; $0,78 \%$ ) was associated with a miscall. However, the probability of a miscall increased with the length of the homopolymer; in particular the 108 homopolymers of 6 or more bp $(1.6 \%$ of total) were associated with 26

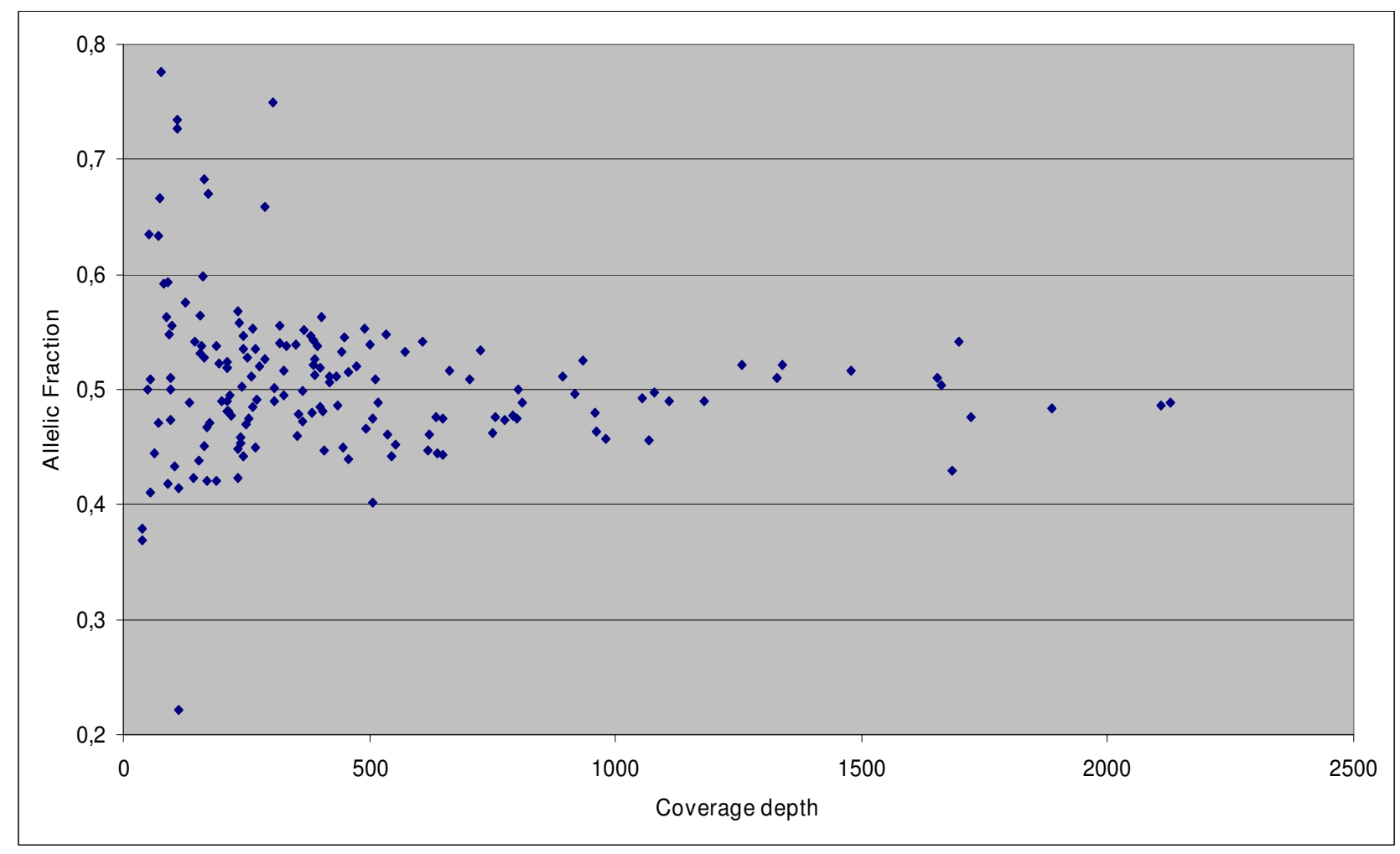

Figure I

Relationship between sequence coverage with the GS-FLX and allelic fraction of heterozygous variations in pool I. 
$(48,1 \%)$ of the 53 miscalls. In contrast, homopolymers were not associated with any false-negative calls: considering sequence positions covered $\geq 30 x$, a total of 132 sequence variants known by Sanger sequencing were contained within homopolymers (Supplementary Table 4 [see Additional file 2]). All were correctly identified.

Finally, since the Blast mapping procedure used in the study was found, in preliminary work, to fail on deletions or insertion longer than $10 \mathrm{bp}$, we devised a separate Blast procedure to analyze GS-FLX reads for these variants. This procedure found no large insertion but, in pool 4, one 44bp heterozygous deletion in exon 49 of Abca4. These findings agree with the variants known by Sanger sequencing.

\section{Discussion}

We assessed the potential of massively parallel pyrosequencing to identify sequence variants in pools of amplicons. Sequencing specificity at reasonable coverage depth $(30 \times)$ was better than expected, with only $0,05 \%$ falsepositive calls. Furthermore, the specificity of detection of SNPs was excellent $(98,5 \%$ for TC calls) whereas errors were mostly related to indels, all lying within homopolymers. False negatives, of primary relevance in diagnostic applications, were absent, provided that $30 \times$ coverage depth was attained.

In order to widen the applicability of our approach, we purposely avoided any primer redesign or primer resynthesis (including primer tails for 454 sequencing as required by the GS-FLX protocol for amplicon sequencing). Tailed oligomers can help in sequencing an amplicon on one side. If both sides are to be sequenced (for increasing reliability and for covering amplicons larger than $250 \mathrm{bp}$ as in this study) one should purchase two new primer couples and run two separate amplifications for each amplicon in the pool. In contrast, library preparation following the conventional 454 sequencing approach (Supplementary Figure 1) yields the required material with little additional time compared to the doubling of amplicon preparation and processing. Indeed, this approach could be of interest to all those who routinely identify sequence variations using any PCR-based technique.

The sample material in this study consisted of amplicons that varied greatly in length, sequence composition and sequence variations. Several of these amplicons were from the same genomic region amplified under the same conditions from different patients, with and without such mutations. We chose this experimental design in order to explore the performance of this approach depending on the molecular complexity under investigation. The pooled DNA samples were subjected to 454 sequencing and the resulting highly redundant representation of the targeted regions was used for blind, bioinformatics identification of sequence variants.

We attempted to normalize the concentrations of amplicons in the pools in order to minimize the variability in the coverage depth among different DNA fragments. However, despite accurate measurements of concentrations before pooling, there was substantial variability in the depth of coverage. Nonetheless, a very high average coverage was attained $(400 \times)$. A relevant fraction of sequence positions (4\%, corresponding to nearly 5 of 110 $\mathrm{Kb}$ ) was below $30 \times$ coverage, thus preventing a reliable call according to our predefined parameters. With less stringent $10 \times$ coverage, $2 \mathrm{~Kb}$ was still below the threshold. On the other hand, nearly $10 \%$ of the sequence had an average coverage depth above $1000 \times$, peaking at over $4000 \times$ in a few cases. These areas of great coverage "waste" a considerable proportion of sequencing power, making this approach less productive than expected and requiring the collection of many more reads than the minimum necessary to exhaustively cover the entire region under scrutiny.

The variability in amplicon coverage may depend on length and GC content, which affect amplification efficiency in emulsion PCR [4]. Alternatively, it may depend on ligation efficiency, which is affected by the sequence of the $3^{\prime}$ and $5^{\prime}$ ends [11]. We found no clear relationship between coverage and either amplicon length or GC content. However, similar problems have been observed in a large resequencing project using the Sanger method, despite the possibility of PCR and sequencing optimization [1]. In a defined diagnostic setting, notwithstanding the reason for such biased (variable) coverage, this problem could be solved by increasing the quantity of "lowyield" amplicons with respect to that of "high yield" ones [7]. After a few trials, normalization conditions could be defined and used for every new sample.

\section{Definition of parameters and identification of sequence variations}

Sequence variations were searched for by comparing actual base calls to the expected sequence known from Sanger sequencing. In preliminary experiments, we evaluated data with $10 \times$ and $30 \times$ coverage depth, and observed that $10 \times$ coverage did not guarantee an allelic fraction (representation of the two alleles) of at least $20 \%$. Therefore, we set our coverage threshold to $30 \times$. To further improve the confidence of the sequencing results, we devised a classification system based on empirical observations. When both strands called the same variation, the call was classified as "top confident" (TC) call; considering only SNPs, only 5 of 340 TC calls were not confirmed; these miscalls were within homopolymers. When just one strand was available for sequence recognition (typically in 
amplicons longer that 250 bases when the variation was close to the amplicon's ends), the variation was classified as "very confident" (VC); only 1 of 44 VC SNP variants was a miscall, again lying within a homopolymer. When there were conflicting results between forward and reverse strands, we gave a "not confident" (NC) classification; this was the case in only 41 base positions. None of these NC calls corresponded to a true variation. Such miscalls were found in amplicons longer than $250 \mathrm{bp}$ and were due to a decrease of sequence quality at the end of the read on one strand. Although this does not seem to be a general problem, when these miscalls occur, they can be simply discarded based on automated classification and visual inspection.

Pyrosequencing is known to be affected by over- or undercalling within homopolymers [12]. Although the combined length of homopolymers totaled nearly $23 \mathrm{~Kb}$, we had just 53 false-positive calls (forty 1-bp deletions, seven 1-bp insertions, and six SNPs) lying within these regions. The miscall rate increased with homopolymer length, up to 9 bp in this study. In continuing experimentation, we have preliminary observations that, in longer homopolymers (e.g. 15- to 20-mers), the length of false-positive deletions also increases beyond single base pairs (unpublished observations). However, since the GS-FLX correctly called 132 sequence variations within homopolymers, these regions should not always be considered unreliable. Nonetheless, deletions and insertions in long homopolymers should be carefully evaluated.

Considering the four pools separately, no clear indication of a specific bias for accuracy was found. However, the smallest pool had the poorest performance in terms of mean coverage.

\section{Evaluation of diagnostic applicability}

False positives (overcalling of variations) were reasonably low: we demonstrated an overall $99,95 \%$ specificity in base calling ( 53 miscalls within $104.7 \mathrm{~Kb}$ of sequence covered $\geq 30 \times)$. All these miscalls were in homopolymeric sequences and more frequently involved deletions and insertions $(n=47)$ rather than SNPs $(n=6)$.

False negatives are a major issue in diagnostics applications. In this study, they were absent among amplicons at $\geq 30 \times$ coverage ( $100 \%$ sensitivity). However, 17 of 429 known sequence variations $(4,0 \%)$ were missed due to insufficient coverage. Decreasing the minimum coverage to $10 \times$ led to the identification of 7 additional variants. Nevertheless, with such a low coverage 7 additional miscalls were added, thus lowering specificity. In addition, a decrease in coverage depth was associated with a greater variability in allelic fraction, making it difficult to reliably call heterozygous variations. Indeed, high redundancy represents one of the major advantages of this approach, allowing for a detailed molecular description of complex mixtures of nucleic acids [13]. Therefore, according to our experience, a minimum $30 \times$ coverage depth is required for reliable detection of variants for diagnostic purposes.

The failure of pyrosequencing to adequately cover certain amplicons may necessitate that standard sequencing be performed on those DNA regions. However, as suggested earlier, in diagnostic laboratories that routinely sequence a defined set of amplicons, countermeasures can be adopted to improve the coverage of amplicons.

\section{Conclusion}

This study confirms the high potential of massively parallel pyrosequencing in the scanning of DNA samples for sequence variations. Compared to traditional sequencing technology, this system is capable of higher throughput and is able to rapidly collect genomic information. Our study highlighted some critical aspects of the technology related to the uniformity of coverage. Based on our observation that the allelic fraction of variants approaches 0.5 as sequence coverage increases from $30 \times$ to over $2000 \times$, in future work coverage depth should be carefully considered, in particular for diagnostic applications; this is a fundamental issue for the reliable detection of heterozygous variants. Moreover, since most sequencing errors were due to indels in homopolymeric tracts, analytical approaches might be specifically developed to better assess these variants. Our results encourage future studies evaluating the diagnostic applications of this sequencing technology in diseases with high allelic and genetic heterogeneity.

\section{Methods \\ DNA samples}

We selected 16 human genes associated with human genetic diseases (Table 1) and obtained corresponding PCR-amplified material from the clinical inventory of San Raffaele Hospital. A total of 343 amplicons were obtained, representing 429 genetic variations already identified by Sanger sequencing. DNA samples had been obtained from patients who had given informed consent or under protocols approved by the hospital's ethics review board.

For all amplicons in the study, PCR had been performed as previously described [14-22] or according to standard procedures (indicated by an asterisk in Table 1). Amplified regions comprised entire exons with intron-exon junctions or exon portions. Amplicons were checked for quality on agarose gel electrophoresis and directly quantified after ethidium bromide staining using a gel scanner (Typhoon 8600, Amersham). 


\section{Amplicon pools}

Amplicons were pooled into 4 samples in equimolar concentrations (Supplementary Table 1 [see Additional file 2]). On several occasions, one amplicon was included in two or more pools. PCR products bearing the same variation were from different individuals, in order to increase the variability under investigation.

Pools were purified using silica spin-columns (MinElute PCR purification kit, Qiagen, Valencia, CA). Each pool contained $2 \mu \mathrm{g}$ DNA determined spectrophotometrically (NanoDrop Technologies, Wilmington, Delaware USA). The pools were checked for quality by capillary electrophoresis (Agilent Bioanalyzer 2100 with the DNA 1000 kit; Agilent Technologies, Palo Alto, CA, USA).

\section{GS-FLX pyrosequencing}

Pools were prepared for FLX sequencing following the Low Molecular Weight DNA protocol as reported in the 454 Roche GS-FLX DNA Library Preparation Kit User Manual. Each pool was separately loaded onto one-fourth of a sequencing plate. Sequencing was performed according to the manufacturer's instructions.

\section{Sequence data analysis}

GS-FLX reads were mapped to reference sequences using Blast v. 2.2.15 [23], with e value set to 1e-7; low complexity filter (DUST) disabled; word size, 4; and -v and -b set to the highest possible number of matches (in a dataset containing 100000 reads, $-\mathrm{v}$ and -b are set to 100000 or higher). Other parameters were left at default. References sequences were those of the NCBI human genome, build 36.3. The Blast output was stored in a PostgreSQL database together with the corresponding read and reference. Using each reference to collect its corresponding read set from the database, the resulting multiple alignment was used to calculate the percentage base call for every sequence position with a coverage depth of 30 (or 10), using a standard query language (SQL) query (available upon request). We defined a sequence variation when unexpected calls (with respect to the reference sequence) exceeded a $20 \%$ allelic fraction (allelic fraction $=$ mutated allele counts/(wildtype allele counts + mutated allele counts)) in regions having $30 \times$ (or 10x) depth coverage. The detailed bioinformatics procedure will be published separately.

Since preliminary work revealed that this Blast procedure was inefficient on deletions and insertions larger than 10 bp, we further processed Blast outputs for reads that did not uniquely match to the reference genome, searching for those matching a single reference (amplicon) sequence twice with proper strandedness.
Sequence variants were automatically classified by an SQL query (available upon request) as follows:

- Top confident (TC): variation called by both forward and reverse reads;

- Very confident (VC): variation called in consensus regions where reads were available from one of the two strands only;

- Not confident (NC): conflicting calls between forward and reverse strands.

To visualize the sequence variants, the SeqMan package (DNASTAR, Madison, WI, USA) was used.

\section{Competing interests}

The authors declare that they have no competing interests.

\section{Authors' contributions}

The study was conceived and coordinated by AA, LRB, MF and GDB, and designed by PC, SBe, LC, MF and GDB. RaB devised the software procedure. RoB and ER generated the 454 sequencing data. SS, AC, CM and Sbo did the Sanger sequencing. Sequence alignment was done by RoB, RaB, ER, PC, SBe, LC, SS, AC, CM, SBo. The manuscript was written by RoB, RaB, ER, MF and GDB.

\section{Additional material}

\section{Additional file 1}

Tables 2, 3,4. Table 2. Composition of four amplicon pools and results of GS-FLX pyrosequencing. Table 3. Sequence variations identified by GSFLX pyrosequencing and comparison to variants known from Sanger sequencing. TC, top confidence; VC, very confident; NC, not confident. Table 4 Homopolymers present in the $110 \mathrm{~Kb}$ DNA sequenced in this study, and relationship to false-positive sequencing calls.

Click here for file

[http://www.biomedcentral.com/content/supplementary/14712164-9-464-S1.doc]

\section{Additional file 2}

Suppl. Fig 1, Suppl Table 1, Suppl Table 2, Suppl Table 3, Suppl Table 4. Supplementary Figure 1. Schematics of the 454 sample processing and sequencing flow. Supplementary Table 1. Pool composition. Supplementary Table 2. Summary of variations. Supplementary Table 3. Comparison of results at $30 \times$ and $10 \times$ coverage. Supplementary Table 4.

Summary of variations in homopolymers.

Click here for file

[http://www.biomedcentral.com/content/supplementary/14712164-9-464-S2.doc]

\section{Acknowledgements}

We gratefully acknowledge the financial support from MIUR FIRB project "Biochip", (RBNEOITZZ8), MIUR FIRB project "MICRAM" 
(RBNE0IZB7A), and MIUR FIRB project "NG-LAB" (RBLA03ER38). Valerie Matarese provided scientific editing.

\section{References}

I. Wood LD, Parsons DW, Jones S, Lin J, Sjöblom T, Leary RJ, Shen D, Boca SM, Barber T, Ptak J, Silliman N, Szabo S, Dezso Z, Ustyanksky V, Nikolskaya T, Nikolsky Y, Karchin R, Wilson PA, Kaminker JS, Zhang Z, Croshaw R, Willis J, Dawson D, Shipitsin M, Willson JK, Sukumar S, Polyak K, Park BH, Pethiyagoda CL, Pant PV, Ballinger DG, Sparks AB, Hartigan J, Smith DR, Suh E, Papadopoulos N, Buckhaults $P$, Markowitz SD, Parmigiani G, Kinzler KW, Velculescu VE, Vogelstein $B$ : The genomic landscapes of human breast and colorectal cancers. Science 2007, 3 I 8: I | 08-13.

2. Sjöblom T, Jones S, Wood LD, Parsons DW, Lin J, Barber TD, Mandelker D, Leary RJ, Ptak J, Silliman N, Szabo S, Buckhaults P, Farrell C, Meeh P, Markowitz SD, Willis J, Dawson D, Willson JK, Gazdar AF, Hartigan J, Wu L, Liu C, Parmigiani G, Park BH, Bachman KE, Papadopoulos N, Vogelstein B, Kinzler KW, Velculescu VE: The consensus coding sequences of human breast and colorectal cancers. Science 2006, 3 I 4:268-274.

3. Chee M, Yang R, Hubbell E, Berno A, Huang XC, Stern D, Winkler J, Lockhart DJ, Morris MS, Fodor SP: Accessing genetic information with high-density DNA arrays. Science I996, 274:6 10-6I4.

4. Shendure J, Porreca GJ, Reppas NB, Lin X, McCutcheon JP, Rosenbaum AM, Wang MD, Zhang K, Mitra RD, Church GM: Accurate multiplex polony sequencing of an evolved bacterial genome. Science 2005, 309:|728-1732.

5. Braslavsky I, Hebert B, Kartalov E, Quake SR: Sequence information can be obtained from single DNA molecules. Proc Natl Acad Sci USA 2003, 100:3960-3964.

6. Margulies M, Egholm M, Altman WE, Attiya S, Bader JS, Bemben LA Berka J, Braverman MS, Chen YJ, Chen Z, Dewell SB, Du L, Fierro JM, Gomes XV, Godwin BC, He W, Helgesen S, Ho CH, Irzyk GP, Jando SC, Alenquer ML, Jarvie TP, Jirage KB, Kim JB, Knight JR, Lanza JR, Leamon JH, Lefkowitz SM, Lei M, Li J, Lohman KL, Lu H, Makhijani VB, McDade KE, McKenna MP, Myers EW, Nickerson E, Nobile JR, Plant R, Puc BP, Ronan MT, Roth GT, Sarkis GJ, Simons JF, Simpson JW, Srinivasan M, Tartaro KR, Tomasz A, Vogt KA, Volkmer GA, Wang SH, Wang Y, Weiner MP, Yu P, Begley RF, Rothberg JM: Genome sequencing in microfabricated high-density picolitre reactors. Nature 2005, 437:376-380.

7. Dahl F, Stenberg J, Fredriksson S, Welch K, Zhang M, Nilsson M, Bicknell D, Bodmer WF, Davis RW, Ji H: Multigene amplification and massively parallel sequencing for cancer mutation discovery. Proc Natl Acad Sci USA 2007, 1 04:9387-92.

8. Porreca GJ, Zhang K, Li JB, Xie B, Austin D, Vassallo SL, LeProust EM, Peck BJ, Emig C], Dahl F, Gao Y, Church GM, Shendure J: Multiplex amplification of large sets of human exons. Nat Methods 2007 , 4:931-6.

9. Albert TJ, Molla MN, Muzny DM, Nazareth L, Wheeler D, Song $X$, Richmond TA, Middle CM, Rodesch MJ, Packard CJ, Weinstock GM, Gibbs RA: Direct selection of human genomic loci by microarray hybridization. Nat Methods 2007, 4:903-5.

10. Hodges E, Xuan Z, Balija V, Kramer M, Molla MN, Smith SW, Middle CM, Rodesch MJ, Albert T], Hannon G], McCombie WR: Genomewide in situ exon capture for selective resequencing. Nat Genet 2007, 39:1522-7.

II. Binladen J, Gilbert MT, Bollback JP, Panitz F, Bendixen C, Nielsen R, Willerslev E: The use of coded PCR primers enables highthroughput sequencing of multiple homolog amplification products by $\mathbf{4 5 4}$ parallel sequencing. PLOS ONE 2007, 2:el 97.

12. Ronaghi $M$, Uhlen $M$, Nyren P: A sequencing method based on real-time pyrophosphate. Science 1998, 28I:363-365.

13. Thomas RK, Nickerson E, Simons JF, Jänne PA, Tengs T, Yuza Y, Garraway LA, LaFramboise T, Lee JC, Shah K, O'Neill K, Sasaki H, Lindeman N, Wong KK, Borras AM, Gutmann EJ, Dragnev KH, DeBiasi R, Chen TH, Glatt KA, Greulich H, Desany B, Lubeski CK, Brockman W, Alvarez P, Hutchison SK, Leamon JH, Ronan MT, Turenchalk GS, Egholm M, Sellers WR, Rothberg JM, Meyerson M: Sensitive mutation detection in heterogeneous cancer specimens by massively parallel picoliter reactor sequencing. Nat Med 2006, 1 2:852-5.

14. Somaschini M, Nogee LM, Sassi I, Danhaive O, Presi S, Boldrini R, Montrasio C, Ferrari M, Wert SE, Carrera P: Unexplained neona- tal respiratory distress due to congenital surfactant deficiency. J Pediatr 2007, I 50:649-53.

15. Stenirri S, Fermo I, Battistella S, Galbiati S, Soriani N, Paroni R, Manitto MP, Martina E, Brancato R, Allikmets R, Ferrari M, Cremonesi L: Denaturing HPLC profiling of the ABCA4 gene for reliable detection of allelic variations. Clin Chem 2004, 50:1336-43.

16. Carrera P, Piatti M, Stenirri S, Grimaldi LM, Marchioni E, Curcio M, Righetti PG, Ferrari M, Gelfi C: Genetic heterogeneity in Italian families with familial hemiplegic migraine. Neurology 1999 , 53:26-33.

17. Zielenski J, Rozmahel R, Bozon D, Kerem B, Grzelczak Z, Riordan JR, Rommens J, Tsui LC: Genomic DNA Sequence of the Cystic Fibrosis Transmembrane Conductance Regulator (CFTR) gene. Genomics 1991, 10:214-228.

18. Cremonesi L, Foglieni B, Fermo I, Cozzi A, Paroni R, Ruggeri G, Belloli S, Levi S, Fargion S, Ferrari M, Arosio P: Identification of two novel mutations in the 5 '-untranslated region of $\mathrm{H}$-ferritin using denaturing high performance liquid chromatography scanning. Haematologica 2004, 88: I I I0-6.

19. Ferrari F, Foglieni B, Arosio P, Camaschella C, Daraio F, Levi S, García Erce JA, Beaumont C, Cazzola M, Ferrari M, Cremonesi L: Microelectronic DNA chip for hereditary hyperferritinemia cataract syndrome, a model for large-scale analysis of disorders of iron metabolism. Hum Mutat 2006, 27:201-8.

20. Foglieni B, Cremonesi L, Travi M, Ravani A, Giambona A, Rosatelli MC, Perra C, Fortina P, Ferrari M: Beta-thalassemia microelectronic chip: a fast and accurate method for mutation detection. Clin Chem 2004, 50:73-9.

2I. Vytopil M, Benedetti S, Ricci E, Galluzzi G, Dello Russo A, Merlini L, Boriani G, Gallina M, Morandi L, Politano L, Moggio M, Chiveri L, Hausmanova-Petrusewicz I, Ricotti R, Vohanka S, Toman J, Toniolo D: Mutation analysis of the lamin A/C gene (LMNA) among patients with different cardiomuscular phenotypes. J Med Genet 2003, 40:el 32.

22. Cremonesi L, Forni GL, Soriani N, Lamagna M, Fermo I, Daraio F, Galli A, Pietra D, Malcovati L, Ferrari M, Camaschella C, Cazzola M: Genetic and clinical heterogeneity of ferroportin disease. $\mathrm{Br}$ J Haematol 2005, I 3 I:663-70.

23. Altschul SF, Gish W, Miller W, Myers EW, Lipman DJ: Basic local alignment search tool. J Mol Biol 1990, 21 5:403-4I0.
Publish with Biomed Central and every scientist can read your work free of charge

"BioMed Central will be the most significant development for disseminating the results of biomedical research in our lifetime. "

Sir Paul Nurse, Cancer Research UK

Your research papers will be:

- available free of charge to the entire biomedical community

- peer reviewed and published immediately upon acceptance

- cited in PubMed and archived on PubMed Central

- yours - you keep the copyright 